\title{
Immune reactivity and host modulatory roles of two novel Haemonchus contortus cathepsin B-like proteases
}

\author{
Mariam Bakshi ${ }^{1,2}$, Wenbin Tuo ${ }^{1}$, Raffi V. Aroian ${ }^{3}$ and Dante Zarlenga ${ }^{1 *}$
}

\begin{abstract}
Background: Haemonchus contortus is a blood-feeding, gastrointestinal nematode (GIN) that causes significant economic losses to the small ruminant industry worldwide. Despite extensive efforts, our understanding of the molecular mechanisms used by GIN to evade host immune responses is limited. Cathepsin B-like proteins (CBPs) are members of the cysteine protease family and are involved in parasite invasion and thus provide viable vaccine candidates.

Methods: In silico comparative analysis was used to identify conserved proteins among a subset of clade $V$ parasitic nematodes with emphasis on blood-feeding worms, among which CBPs appeared prominently. We identified and characterized two novel CBPs designated Hc-CBP-1 and Hc-CBP-2. Rabbit anti-recombinant (r) Hc-CBP-1 and rHcCBP-2 were used to detect the presence of native proteins in the excretory secretory products (ESP) and in worm tissues of adult $\mathrm{H}$. contortus. Peptide arrays of $\mathrm{rHc}-\mathrm{CBP}-1$ and $\mathrm{rHc}-\mathrm{CBP}-2$ were screened with the homologous and heterologous anti-sera and with sera from dexamethasone-treated $\left(\mathrm{Dex}^{+}\right)$and non-treated $\left(\mathrm{Dex}^{-}\right)$H. contortus-infected animals to identify key immunogenic peptides. Gene transcription of Hc-cbp-1 and $\mathrm{Hc}-c b p-2$ was also performed on H. contortus-infected animals treated with Dex ${ }^{+}$. Finally, the mature recombinant proteins were used to assess their abilities to modulate cell functions.

Results: Immunohistochemistry showed that both $\mathrm{Hc}-\mathrm{CBP}-1$ and $\mathrm{Hc}-\mathrm{CBP}-2$ are present on the brush borders of the intestine; $\mathrm{Hc}-\mathrm{CBP}-2$ was also present in the hypodermis of the body wall. Peptide displays screened with rabbit anti-rHc-CBP-1 and anti-rHc-CBP-2 revealed regions within the proteins where dominant and overlapping epitopes prevailed. ELISA results were consistent with only Hc-CBP-1 being present in $\mathrm{H}$. contortus adult ESPs. H. contortus from Dex ${ }^{+}$animals exhibited a threefold increase in $\mathrm{Hc}-c b p-2$ transcript while $\mathrm{Hc}-\mathrm{cbp}-1$ expression did not change. In contrast, comparisons of immunoreactivities of $\mathrm{rHc}-\mathrm{CBP}-1$ and $\mathrm{rHc}-\mathrm{CBP}-2$ peptide arrays to sera from Dex ${ }^{+}$and Dex $^{-}$ animals primarily showed changes in Hc-CBP-1 binding. Lastly, rHc-CBP-1 suppressed mRNA expression of bovine peripheral blood mononuclear cell cytokines/activation markers, including TNFa, IL-1, IL-6 and CD86.
\end{abstract}

Conclusions: These results suggest that as secreted and cryptic proteins, respectively, Hc-CBP-1 and Hc-CBP-2 influence cellular and immunological activities that have interesting dynamics during infection and may provide viable immune-related targets for attenuating H. contortus infectivity.

Keywords: Haemonchus contortus, Gastrointestinal, Cathepsin B, Cysteine proteases, Peripheral blood mononuclear cells

${ }^{*}$ Correspondence: dante.zarlenga@usda.gov

${ }^{1}$ Animal Parasitic Diseases Laboratory, USDA-ARS, Beltsville, MD, USA

Full list of author information is available at the end of the article

\section{Background}

Gastrointestinal nematode (GIN) infections are ranked among the most economically important diseases of 
livestock worldwide [1]. Haemonchus contortus is the most pathogenic of this group in small ruminants because both fourth-stage larvae (L4) and mature adult worms feed from capillaries in the abomasum. Infections are often accompanied by hemorrhagic gastritis, hypoproteinemia, anemia and edema, where acute infections can cause death [2]. A single adult worm is estimated to cause $30-50 \mu \mathrm{l}$ of blood loss per day [3]. Overuse and abuse of anthelmintics have resulted in resistance to all major drug classes [4]. This, in conjunction with chemical residues in animal products, has advanced the need for alternative and sustainable control methods. Vaccination remains a viable albeit difficult option because it targets both resistant and susceptible strains of the parasite.

The design of effective vaccines is underpinned by advancing our understanding of host-parasite interactions, the immune responses involved in protection and the biology of the worm. While in the abomasum, parasite excretory secretory products (ESPs) are released that interact with the host to facilitate the infection process and modulate host immune responses [5-7]. Helminth infection can be accompanied by a disruption of the abomasa mucosa and hyperplasia of numerous cell lineages including epithelial cells [8], mast cells [9], lymphocytes [10-12] and eosinophils [13, 14]. To date, both H. contortus ESP ( $\mathrm{Hc}$-ESP) and digestive enzyme complexes have been tested for vaccine potential [15-19]. Some of these antigens constitute the native Barbervax ${ }^{\mathrm{TM}}$ vaccine, which is based upon a cryptic, gut-derived antigen preparation, H-Gal-GP, which consists of pepsins, metalloproteases, cysteine protease-like enzymes and $\mathrm{H} 11$, an integral membrane glycoprotein complex [20-22]. This vaccine is commercially available but sold to a limited market in Australia, New Zealand and South Africa because it is naturally derived from experimentally infected animals which constrains production and distribution. Unfortunately, protection is transient, requiring revaccination every 6 weeks. Recombinant DNA technology is one way to circumvent production issues, but this has been largely unsuccessful thus far [23].

The hematophagous lifestyle of $H$. contortus adult worms has armed them with an array of molecules essential for feeding such as cathepsin B-like proteases that digest hemoglobin, serum proteins and host cells [24]. Cathepsin B family members have been described in $H$. contortus that are important in pathogenesis; however, embryogenesis, molting, egg hatching and yolk degradation are additional functions associated with the protein family members making them viable targets for vaccine development $[25,26]$. In other parasites like Fasciola hepatica, cathepsin B interferes with host immunity linked to peripheral blood mononuclear cells (PBMC) and induces pro-inflammatory responses [27]. During acute infections, cathepsin B from Schistosoma mansoni stimulates mixed Th1/Th2/Th17 immune reactions in mice resulting in a transient Th17 response [28]. Collectively, these and other data are consistent with an association between considerable immunogenic capacity and cathepsin B-like proteases.

Amidst extensive efforts to identify vaccine targets, studies are needed to better characterize deeper structural and immune-modulatory capacities imposed by $H$. contortus cathepsin B-like proteases and their roles as virulence factors. In the present study, two $H$. contortus cathepsin B-like cysteine proteases (CBP), Hc-CBP-1 and $\mathrm{Hc}-\mathrm{CBP}-2$, were identified using in silico analysis of select parasitic clade $\mathrm{V}$ nematodes and then biochemically characterized. Peptide assays were used to investigate immunogenic regions of recombinant ( $r$ ) Hc-CBP-1 and $\mathrm{rHc}-\mathrm{CBP}-2$. The roles of these proteins in modulating host PBMCs, cytokine production and co-stimulatory surface marker expression were examined. We suggest that rHc-CBP-1 and rHc-CBP-2 are effective immunogens and hold promise as vaccine candidates against haemonchosis.

\section{Methods \\ Identification of Hc-CBP-1 and Hc-CBP-2 by in silico analyses}

Amino acid sequences for proteases and protease inhibitors from parasitic clade $\mathrm{V}$ nematodes $[H$. contortus (Additional file 1: Dataset S1), Teladorsagia circumcincta, Necator americanus, Ancylostoma caninum)], non-parasitic nematodes (Caenorhabditis elegans and Pristionchus pacificus) and domestic sheep, Ovis aries, were obtained from NCBI and MEROPS databases. Putative secreted proteases were identified by the presence of amino-terminal signal sequences as predicated by SignalP 5.0 (http:// www.cbs.dtu.dk/services/SignalP) algorithms. Family classification of protein sequences was determined from the conserved domain database in NCBI (CDD) [29].

Sequences from each species were subjected to local BLASTp (CLC Genomics Workbench, Qiagen, Hilden, Germany) with a BLOSUM62 matrix and an expectation value of 10.0. Sequences exhibiting $>60 \%$ similarity among $H$. contortus, C. elegans, $P$. pacificus and $O$. aries were excluded from further analysis. All other sequences from the T. circumcincta, $N$. americanus and $A$. caninum databases were aligned in pairwise fashion with the $H$. contortus database using local BLAST. This resulted in a subset of aspartate and cysteine proteases that were common among all databases. From the list of cysteine proteases, two $H$. contortus cathepsin B-like proteases, designated as Hc-cbp-1 and Hc-cbp-2 for gene and HcCBP-1 and Hc-CBP-2 for protein, were selected for further analysis. 


\section{Parasite collection}

All experimental protocols involving animals were approved by the Beltsville Animal Care and Use Committee and performed in accordance with relevant guidelines and regulations. Male Suffolk sheep and Holstein calves, 3-6 months of age, were inoculated with $\sim 5000$ and $\sim 50,000 \mathrm{H}$. contortus infective L3, respectively, which were obtained by culturing feces, then purified by floatation in a lymphocyte separation medium and washed in $1 \times$ phosphate buffer saline (PBS), or from feces containing eggs that were cultured for 2 weeks in a moistened mixture of vermiculite at $25{ }^{\circ} \mathrm{C}$ and then filtered twice through a Baermann funnel [30]. For adult worms, calves were killed at 21 days post infection (dpi). Adults were purified from abomasal contents by migration from agar slabs as described [30] and then washed three times with $1 \times$ PBS containing $1 \times$ penicillin/streptomycin and glutamine supplement. To collect $H$. contortus excretory secretory products (Hc-ESP), sheep were killed no less than 30 days post infection; 95\% of adult worms were recovered, washed and cultured for $24 \mathrm{~h}$ in $1 \times$ Dulbecco's modified essential medium (DMEM) (Lonza, Basel, Switzerland) supplemented with glutamine, $1 \times$ penicillin/streptomycin and gentamicin as described [31]. The culture supernatant was concentrated using Amicon spin columns (Millipore Sigma, Bedford, MA) and Pierce ${ }^{\mathrm{TM}}$ protein concentrators (Life Technologies, Carlsbad, CA). The purified Hc-ESPs were separated by electrophoresis on an $8-16 \%$ SDS-PAGE gel (Genscript, Piscataway, NJ) under reducing conditions and visualized by Coomassie blue stain. The remaining adult worms were fixed in $10 \%$ neutral formalin and used for immunohistochemical staining.

\section{Dexamethasone drug treatment in $\mathrm{H}$. contortus-infected sheep}

Six Suffolk sheep were maintained in a parasite-free environment with continuous feed and water according to an approved animal care protocol. Two groups of three animals were infected with $5000 \mathrm{H}$. contortus L3. One group $\left(\mathrm{Dex}^{+}\right)$was administered $10 \mathrm{ml}$ of $2 \mathrm{mg} / \mathrm{ml}$ dexamethasone in the hind quarters on 21, 25, 29, 32 and $35 \mathrm{dpi}$ [32]. The control group $\left(\mathrm{Dex}^{-}\right)$was given a similar volume of phosphate-buffered saline. On days 37, 38 and 39, animals were killed; heparinized blood and adult worms were collected. Serum was separated from the blood for ELISA, and adult worms were collected for transcript expression of Hc-cbp-1 and Hc-cbp-2 by quantitative PCR as described below.

\section{Structural analysis}

The 3D structures of Hc-CBP-1 and Hc-CBP-2 were modeled in silico with the SWISS-MODEL protein structure homology modeling server [33]. The best template for each 3D structure was selected using the SWISS-MODEL template library search. The optimality of the predicted structures was estimated using global model quality estimation (GMQE) and qualitative model energy analysis (QMEAN) [34] where GMQE combines the properties from the target-template alignment and the template search methods. The obtained structures were validated using Ramachandran plots [35]. The structures with a higher number of residues in favored regions (>90\%) and fewer residues in outlier regions were selected.

Recombinant protein expression of Hc-CBP-1 and Hc-CBP-2 Total RNA was isolated from $H$. contortus adult worms homogenized in TRIzol ${ }^{\circledR}$ reagent (Thermo Fisher Scientific, Carlsbad, CA). Crude RNA was DNAse treated, column purified (Zymo Research, Irvine, CA) and eluted with nuclease-free water. Template cDNA was synthesized using an cDNA synthesis kit as recommended by the manufacturer (Thermo Fisher Scientific, Carlsbad, CA). The cDNAs were PCR amplified using forward and reverse primers with restriction sites, EcoRI and NotI, for Hc-CBP-1 and SalI and NotI for Hc-CBP-2 (Table 1), and the amplified products were inserted into the pCR2.1 cloning vector (Thermo Fisher Scientific, Carlsbad, CA) by TA cloning. Isolated plasmids were restriction enzyme digested and the inserts subcloned into the expression vector pET29b $(+)$ containing a hexa-histidine tag for downstream protein purification. Plasmids containing subcloned fragments were used to transform E. coli BL21 for protein expression, and the reading frames of the final products were validated by automated sequencing.

Following sequence verification, overnight cultures of each clone were transferred to $500 \mathrm{ml} \mathrm{LB}$ containing $50 \mu \mathrm{g} / \mathrm{ml}$ kanamycin and then grown at $37{ }^{\circ} \mathrm{C}$ until the $\mathrm{OD}_{600}=0.5-0.7$. Isopropyl $\beta$-D-1thiogalactopyranoside was added to a final concentration of $0.5 \mathrm{mM}$ to induce protein expression, and the incubations were continued at $16{ }^{\circ} \mathrm{C}$ for $20 \mathrm{~h}$. The cells were collected by centrifugation at $10,000 \times g$ for $10 \mathrm{~min}$ and the pellets sonicated in lysis buffer (50 $\mathrm{mM} \mathrm{NaH}_{2} \mathrm{PO}_{4}, \mathrm{pH} 7.4 ; 300 \mathrm{mM} \mathrm{NaCl}$, and $10 \mathrm{mM}$ imidazole). The lysates were centrifuged at $12,000 \times g$ for $20 \mathrm{~min}$ and the cleared supernatants passed over Ni-NTA agarose affinity columns (Macherey-Nagel, Düren, Germany) and eluted in 5-ml increments with elution buffers containing imidazole gradient (10 mM-400 mM). The purified fractions were analyzed in SurePAGE ${ }^{\mathrm{TM}}$ (Genscript, Piscataway, NJ) 4-20\% BIS-TRIS gradient gel and stained by Coomassie stain. Samples containing the highest amount of 
Table $1 \mathrm{Hc}-\mathrm{cbp}-1$ and $\mathrm{Hc}-\mathrm{cbp}-2$ recombinant protein expression and qPCR primers

\begin{tabular}{|c|c|c|}
\hline Gene name & $\begin{array}{l}\text { Primer sequence } \\
\text { Bold and underlined: restriction sites }\end{array}$ & $\begin{array}{l}\text { Recombinant protein } \\
\text { expression (E) or } \mathrm{qPCR}(\mathrm{Q}) \\
\text { primers }\end{array}$ \\
\hline Hc-CBP-1 & $\begin{array}{l}\mathrm{F}: \underline{\text { GAATTCTACGGAAGTACTTGGGGATTCTA }} \\
\mathrm{R}: \underline{\text { GCGGCCGCTAGTCTTCTAGTCACGTCGTT }}\end{array}$ & E \\
\hline Hc-CBP-2 & $\begin{array}{l}\text { F: GTCGACCAAGCGGATGTGCTCGCTGCTT } \\
\text { R: } \underline{\text { GCGGCCGCCCCTTGAACATGCCCGGCGA }}\end{array}$ & E \\
\hline $\mathrm{Hc}-c b p-1$ & $\begin{array}{l}\text { F: ACAAGTTCTACGGCAAGGGA; } \\
\text { R: GCGGTGTGTTTGTAGACTCC }\end{array}$ & Q \\
\hline Hc-cbp-2 & $\begin{array}{l}\text { F: GCGAGGACGCTTACGAATTA; } \\
\text { R: GGAGAAGTCCTCGTAAACAGTG }\end{array}$ & Q \\
\hline
\end{tabular}

recombinant protein in the absence of non-specific binding were pooled and concentrated. Protein concentrations were determined using a bicinchoninic acid (BSA) quantification kit (Thermo Fisher Scientific, Carlsbad, CA). Rabbit polyclonal antibodies against purified rHc-CBP-1 and rHc-CBP-2 were generated by Thermo Fisher Scientific (Carlsbad, CA) and validated by Western blot analysis. Prior to downstream studies, endotoxin was removed from rHc-CBP-1 and rHc-CBP-2 preparations using Triton X-114 [31] with final concentration of $1 \%$ and then vortexed for $10 \mathrm{~s}$. The homogeneous mixture was then incubated on ice for $5 \mathrm{~min}$, warmed to $37^{\circ} \mathrm{C}$ for $10 \mathrm{~min}$ to promote phase separation and then spun for $10 \mathrm{~min}$. The upper aqueous layer was transferred to a fresh tube, and the process was repeated. Endotoxin levels were evaluated using an endotoxin detection kit (Limulus Amoebocyte Lysate Kit, Qiagen, Hilden, Germany).

\section{Detection of native Hc-CBP-1 and HC-CBP-2 in H. contortus adults and Hc-ESPs}

Adult crude worm extracts and Hc-ESPs were quantified and diluted to $1 \mu \mathrm{g}$ in $100 \mu \mathrm{l}$ in ELISA coating buffer (sodium bicarbonate buffer, $\mathrm{pH}$ 9.0) and then loaded onto 96-well plates for overnight incubation at $4{ }^{\circ} \mathrm{C}$. The plates were blocked with $0.05 \%$ Tween-20 and $1 \times$ PBS (PBS-Tween) containing 5\% bovine serum albumin (BSA) and then incubated with polyclonal antibodies against rHc-CBP-1 or rHc-CBP-2 (1:200) in $1 \times$ PBS containing $5 \%$ BSA. After washing, the wells were incubated with horseradish peroxidase (HRP) conjugated goat anti-rabbit secondary antibody (1:1000) (Thermo Fisher Scientific, Carlsbad, CA) for $1 \mathrm{~h}$ and then washed with PBS-Tween-20. Substrate (ABTS; 2,2'-azino-bis (3-ethylbenzothiazoline-6-sulfonic acid) was added, and the reaction was stopped using $1 \%$ sodium dodecyl sulfate (SDS). Plates were read at $410 \mathrm{~nm}$ using a SpectraMax spectrophotometer (Molecular Devices, San Jose, CA). All samples were assayed in triplicate.
Immunolocalization of native Hc-CBP-1 and Hc-CBP-2 in $\mathrm{H}$. contortus adult worms

Haemonchus contortus adult worms were collected from the abomasa of infected animals, fixed in $10 \%$ neutral formalin, embedded in paraffin and sectioned at $5 \mu \mathrm{m}$ thickness (HistoServe Inc., Germantown, MD, USA). The slides were deparaffinized and rehydrated through xylene/ethanol washes, then quenched with $3 \% \mathrm{H}_{2} \mathrm{O}_{2}$ and rehydrated prior to antigen recovery in $0.4 \%$ pepsin $1 \%$ hydrochloric acid at $37{ }^{\circ} \mathrm{C}$ for $15 \mathrm{~min}$. The sections were washed twice with $0.75 \%$ of $30 \%$ BRIJ-35 (Millipore Sigma, Burlington, MA) in $1 \times$ PBS (BRIJ-PBS), blocked with $0.5 \%$ sodium caseinate in BRIJ-PBS for 10 min and then incubated with rabbit antisera to $\mathrm{rHc}-\mathrm{CBP}-1$ or $\mathrm{rHc}-$ CBP-2 (1:800 dilution) and pre-immune sera (1:800 dilution) for $30 \mathrm{~min}$ at room temperature. After washing, the slides were incubated with goat anti-rabbit HRP conjugated secondary antibody (GARP) for $20 \mathrm{~min}$ and treated with 3,3' diaminobenzidine (DAB) substrate (Abcam, Cambridge, MA). Hematoxylin and HistoMark TrueBlue (VWR, Radnor, PA) were used as counterstains. Micrographs were generated using the Zeiss Axioskiope 2 Plus microscope (Zeiss, Thornwood, NY, USA).

\section{Quantitative PCR of Hc-cbp-1 and Hc-cbp-2 in H. contortus derived from $\mathrm{Dex}^{+}$and $\mathrm{Dex}^{-}$injected animals}

Five $H$. contortus adult worms were hand-selected from each animal, homogenized in TRIzol and then used for total RNA isolation as per the manufacturer's instructions. Quantitative PCR (qPCR) was performed in triplicate from synthesized cDNA (1:10 dilution) in $25 \mu \mathrm{l}$ volume containing $300 \mathrm{nM}$ each (Table 1 ) and $2 \times$ SYBR green PCR master mix (Bio-Rad, Hercules, CA) as follows: $50{ }^{\circ} \mathrm{C}$ for $2 \mathrm{~min}, 95^{\circ} \mathrm{C}$ for $10 \mathrm{~min}$, then 40 cycles of $95{ }^{\circ} \mathrm{C}$ for $15 \mathrm{~s}$ and $60{ }^{\circ} \mathrm{C}$ for $1 \mathrm{~min}$. Primer efficiencies were tested using melting curve analysis (Bio-Rad, Hercules, CA). Hc-cbp-1- and Hc- $c b p$-2-specific qPCR primers were selected and validated from aligned sequences and generated fragments of $121 \mathrm{bp}$ and $104 \mathrm{bp}$, respectively. 
The mRNA expression levels for Hc-cbp-1 and Hc-cbp-2 were determined using the $\Delta \Delta \mathrm{Ct}$ method [36]. Glyceraldehyde 3-phosphate dehydrogenase (GAPDH) was used as a housekeeping gene for normalization [37].

\section{Hc-CBP-1 and Hc-CBP-2 peptide arrays}

Forty-six peptides, 15 amino acids (aa) in length, were synthesized (New England Peptide, Garner, MA) with an 8-aa overlap among adjacent peptides (Additional file 6: Dataset S6) and collectively spanned the lengths of each mature protein [Hc-CBP-1 (329 aa) and Hc-CBP-2 (328 aa)]. Peptides encompassing putative signal peptide regions were not included. Each peptide was reconstituted in $200 \mu \mathrm{l}$ dimethyl formamide. Each peptide $(10 \mu \mathrm{l})$ in a total volume of $200 \mu \mathrm{l}$ coating buffer was plated in duplicate and incubated overnight at $4{ }^{\circ} \mathrm{C}$. Peptides were incubated overnight at $4{ }^{\circ} \mathrm{C}$ with rabbit polyclonal antibodies to rHc-CBP-1-, rHc-CBP-2- (1:1000) and H. contortus-infected sheep sera $(1: 1000)$. Sheep sera $(1: 250)$ from $H$. contortus-infected and Dex ${ }^{+}$- and $D^{-} x^{-}$-treated sheep were also incubated overnight at $4{ }^{\circ} \mathrm{C}$ with each peptide array separately. All wells were washed with $1 \times$ PBS-Tween and then incubated with either goat antirabbit HRP conjugated secondary antibody (1:1000) or goat anti-sheep IgG-HRP conjugated antibody (1:1000) at room temperature for $1 \mathrm{~h}$. Colorimetric detection was performed using ABTS substrate after the addition of $1 \%$ SDS to terminate the reactions. Positive binding was visualized at $410 \mathrm{~nm}$ using a SpectraMax spectrophotometer (Molecular Devices, San Jose, CA). Each of these arrays was repeated twice and done in duplicate and triplicate.

\section{PBMC activation assay using rHc-CBP-1}

Bovine blood was collected into sodium citrate buffer using Vacutainers ${ }^{\circledR}$ (Beckton Dickinson, Franklin Lakes, NJ). Cells (PBMC), isolated by density gradient centrifugation in Ficoll-Paque (GE healthcare, Chicago, IL), were washed with balanced salt solution (BSS; $5.5 \mathrm{mM}$ anhydrous glucose, $0.05 \mathrm{mM} \mathrm{CaCl}, 1.0 \mathrm{mM} \mathrm{MgCl}$, $5.4 \mathrm{mM} \mathrm{KCl}, 145 \mathrm{mM}$ TRIS, and $140 \mathrm{mM} \mathrm{NaCl}, \mathrm{pH}$ 7.4) then stained with trypan blue to assess viability. Purified PBMCs $\left(5 \times 10^{4}\right)$ were seeded onto 24-well tissue culture plates in $1 \mathrm{ml}$ DMEM and treated with $0.1,1$ or $10 \mu \mathrm{g} /$ $\mathrm{ml}$ of endotoxin-free rHc-CBP-1 or PBS (control). Native Hc-CBP-1 was not observed in Hc-ESP and therefore was not tested. The cathepsin B inhibitor CA-074 (Millipore Sigma., Burlington, MA) was used to suppress cathep$\sin B$ protein activity in the presence of $0.1,1$ or $10 \mu \mathrm{g} /$ $\mathrm{ml}$ endotoxin-free rHc-CBP-1. Lipopolysaccharide (LPS) (kindly provided by Dr. Harry Dawson, USDA, Beltsville, $\mathrm{MD}$ ) and Concanavalin A (Con A) were used separately as positive controls. The culture plates were incubated at $37{ }^{\circ} \mathrm{C}$ with $5 \% \mathrm{CO}_{2}$ for $72 \mathrm{~h}$. The cells were collected for total RNA isolation and cDNA synthesis as described above. Quantitative PCR (qPCR) was performed in triplicate from cDNA (1:10 dilution) in $25 \mu$ l containing $300 \mathrm{nM}$ each of primer [IL-1, IL-6, tumor necrosis factor (TNF) $\alpha, C D 40, C D 80$ and CD86] [30] and $2 \times$ SYBR green PCR master mix (Bio-Rad, Hercules, CA) as follows: $50{ }^{\circ} \mathrm{C}$ for $2 \mathrm{~min}, 95^{\circ} \mathrm{C}$ for $10 \mathrm{~min}$, then 40 cycles of $95{ }^{\circ} \mathrm{C}$ for $15 \mathrm{~s}$ and $60{ }^{\circ} \mathrm{C}$ for $1 \mathrm{~min}$. Cognate mRNA expression levels were determined using the $\Delta \Delta \mathrm{Ct}$ method [36] using GAPDH as a housekeeping gene for normalization [37]. Primer efficiencies were validated using melting curve analysis (Bio-Rad, Hercules, CA).

\section{Statistical analysis}

Quantitative PCR results were normalized to expression values obtained from $H$. contortus adults from $\mathrm{Dex}^{-}$animals. One-way analysis of variance followed by Tukey's and/or Dunnett's post hoc test was done to compare preimmune and immunized animals and relative fold changes in transcription between $\mathrm{Dex}^{+}$and $\mathrm{Dex}^{-}$animals. Twotailed pairwise $t$-test was done to compare peptide arrays screened with sera from $\mathrm{Dex}^{+}$and $\mathrm{Dex}^{-}$animals. The antibody-binding signal intensities between animals were normalized to mean absorbance signal intensities of each group across the entire array using the formula: absorbance/mean absorbance of two animals $\times 100$. The data are represented as means \pm standard error (SE) where $p \leq 0.05$ is considered statistically significant using Prism 8.4.1 (Graph Pad Software Inc, San Diego, CA).

\section{Results}

\section{In silico discovery and 3D modeling of HC-CBP-1} and Hc-CBP-2

Using the culling mechanisms described in Fig. 1, $H$. contortus sequences that showed $<60 \%$ identity with $O$. aries, $P$. pacificus and C. elegans were selected (Additional file 2: Dataset S2). From this list, 114, 106 and 145 sequences from $H$. contortus exhibited $>60 \%$ identity with sequences from $T$. circumcincta, A. caninum and $N$. americanus, respectively (Additional file 3: Dataset S3). Among these, 69 proteases were present in all four species (Additional file 3: Dataset S3 and Additional file 4: Dataset S4); two were defined as cathepsin B-like cysteine proteases (Additional file 4: Dataset S4 and Additional file 5: Dataset S5) and designated Hc-cbp-1 (GenBank accession no. CDJ83387.1) and Hc-cbp-2 (GenBank accession no. CDJ87123.1). The predicted rHc-CBP-1 and rHc-CBP-2 protein sequences were 349 and 346 amino acids in length with theoretical molecular masses of 39.56 and $38.57 \mathrm{kDa}$, respectively. 
Haemonchus contortus protein sequences were retrieved from the MEROPS and NCBI databases (Additional file 1: Dataset 1)

Redundant sequences were removed, and remaining sequences classified using conserved domain and signal peptide analyses

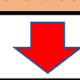

Local BLASTp of $H$. contortus cysteine and aspartate proteases was performed with peptidase databases from non-parasitic nematodes Priscionchus pacificus and Caenorhabitis elegans, host Ovis aries (Additional file 2: Dataset 2) and parasitic nematodes Necator americanus, Ancylostoma caninum and Teledorsagia circumcincta (Additional file 3: Dataset 3) using CLC genomics workbench ${ }^{\circledR}$

Aspartate and cysteine protease sequences were selected that possess less than $60 \%$ similarity with peptidases from $P$. pacificus, $C$. elegans and host $O$. aries, and greater than $60 \%$ similarity with $N$. americanus, $A$. caninum and $T$. circumcincta (Additional file 4: Dataset 4)

Haemonchus contortus aspartate and cysteine proteases were selected and verified by conserved domain analysis (Additional file 5: Dataset 5)

Cathepsin B like proteases Hc-cbp-1 (GenBank Accession no. CDJ83387.1) and Hc-cbp-2 (GenBank Accession no. CDJ87123.1) were selected for further characterization

Fig. 1 In silico analysis for identifying H. contortus cathepsin B-like proteases, Hc-CBP-1 and Hc-CBP-2. H. contortus proteases were selected from NCBI and MEROPS databases and subjected to local BLASTp against clade $V$ parasitic helminths
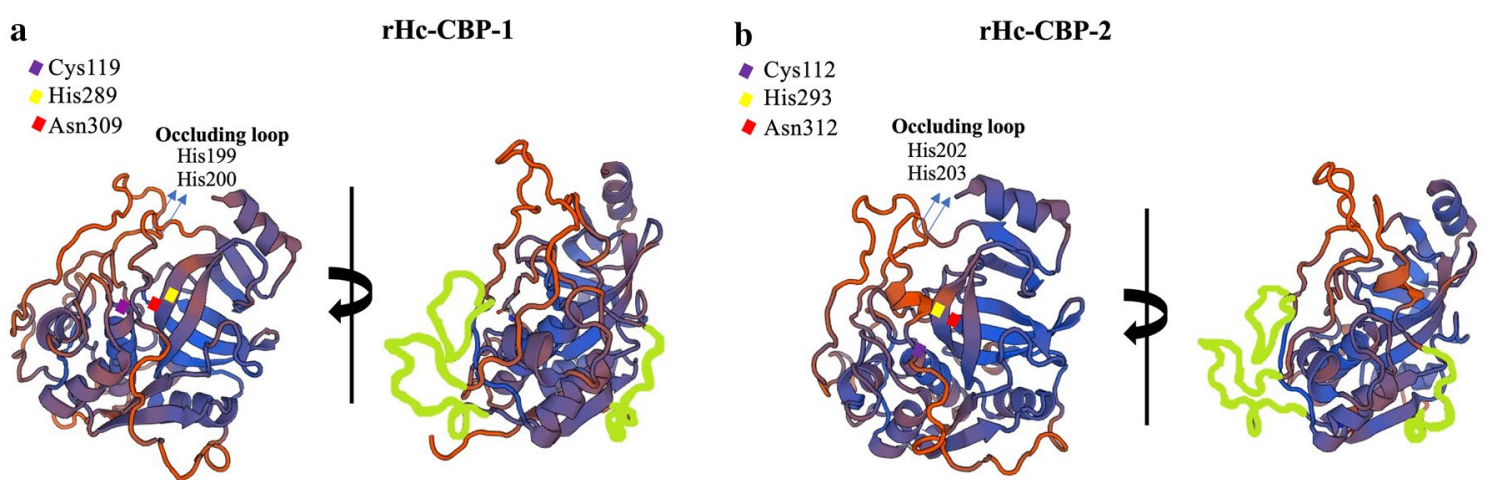

Fig. 2 Three-dimensional modeling of H. contortus cathepsin B-like proteases Hc-CBP-1 and Hc-CBP-2. The 3D models of Hc-CBP-1 and Hc-CBP-2 were generated using the $x$-ray crystallographic structure of mature cathepsin B from the genus Rattus using SWISS-MODEL. The catalytic triad residues Cys119 (a) and Cys112 (b) were in the left domain and His289 (a) and His293 (b) and Asn309 (a) and Asn312 (b) in the right domain. On the right panel, the surface-exposed loops specific to H. contortus are highlighted in green. The residues delimitating the loops-Leu73-Pro80, Asn182-Pro193, Arg221-Asp233 for Hc-CBP-1 and between Arg-74-Asp89, Tyr186-Pro194 and Cys221-Thr238 for Hc-CBP-2-are highlighted in green using Microsoft Paint (Microsoft Corporation, Redmond, WA)

The putative structures of native Hc-CBP-1 and HcCBP-2 were determined using the SWISS-MODEL [36] and the catalytic domain of the mature Rat cathepsin B, which shares $46 \%$ and $43 \%$ sequence identity with rHcCBP-1and rHc-CBP-2, respectively. Both rHc-CBP-1 and rHc-CBP-2 exhibit the typical papain-like fold that is characteristic of members of the cathepsin family (Clan CA, family C1) and are composed of left (L) and right (R) domains [38] (Fig. 2). The left domain has three helical regions, and the right domain is composed of a barrel of six strands, which includes a shorter $\alpha-$ helical motif where the catalytic triad is housed in a 
Presence in $\mathrm{Hc}$-ESP

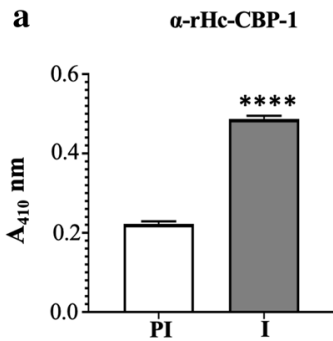

b

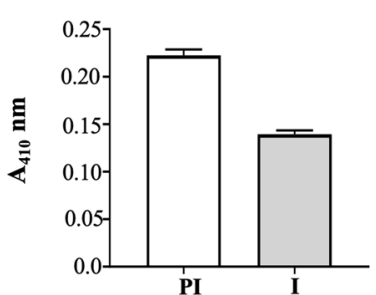

Presence in $\mathrm{Hc}$ crude extract

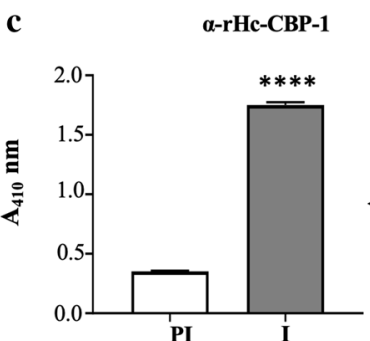

d

$\alpha-$-Hc-CBP-2

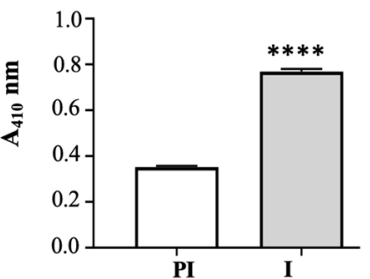

Fig. 3 Detection of native Hc-CBP-1 and Hc-CBP-2 in adult Hc-ESP and crude worm extract. ELISA was used to detect Hc-CBP-1 and Hc-CBP-2 in Hc-ESP (a) and adult crude worm extract (b) using anti a-rHc-CBP-1 (a and $\mathbf{c}$ ), a-rHc-CBP-2 (b and d) and pre-immune serum (1:200 dilution). Data are reported as the mean $(M)$ of triplicates \pm SEM captured at Absorbance of $410 \mathrm{~nm}\left(\mathrm{~A}_{410}\right)$. ${ }^{* * *} p \leq 0.0001$ indicates statistical significance between pre-immune $(\mathrm{PI})$ and immune (I) serum samples. NS means not significant

cleft separating the two domains. The 3D models of rHc-CBP-1 and rHc-CBP-2 show that the two domains containing Cys119 (Fig. 2a) and Cys112 (Fig. 2b) are in the left domain, and His289 (Fig. 2a), His293 (Fig. 2b), Asn309 (Fig. 2a) and Asn312 (Fig. 2b) are in the right domain. Both structures exhibit the canonical occluding loop between the conserved Cys197 and Asp214 residues in Hc-CBP-1 (Fig. 2a) and the Cys200 and Asp215 in Hc-CBP-2 (Fig. 2b) and are further characterized by two adjacent histidine residues (His199 and His200 for rHc-CBP-1 and His202 and His203 for rHcCBP-2) that putatively block the active site cleft responsible for the dipeptidyl carboxypeptidase activity. The major structural differences in backbone superposition correspond to additional residues and are localize to the surface-exposed loops, which include residues Leu73-Pro80, Asn182-Pro193 and Arg221-Asp233 for Hc-CBP-1 and Arg74-Asp89, Tyr186-Pro194 and Cys221-Thr238 for Hc-CBP-2.

\section{Expression of rHc-CBP-1 and rHc-CBP-2 and detection of native proteins}

The full-length cDNA sequences encoding recombinant (r) rHc-CBP-1 and rHc-CBP-2 are identical to those in the GenBank database where expression was achieved in bacterial expression system (Additional file 7: Figure S1) which were used to generate polyclonal antibodies. Using polyclonal rabbit antibodies to $\mathrm{rHc}-\mathrm{CBP}-1$ and rHC-CBP-2, the native Hc-CBP-1 was found to be highly expressed in the Hc-ESP (Fig. 3a), whereas Hc-CBP-2 was undetectable in the Hc-ESP by ELISA (Fig. 3b). However, both Hc-CBP-1 (Fig. 3c) and Hc-CBP-2 (Fig. 3d) were present at high levels in crude extracts of adult worm. Immune reactivity of the $\mathrm{rHc}-\mathrm{CBP}-1$ antiserum to adult worm extracts was significantly stronger than with HcCBP-2 antiserum (Fig. 3c, d).
Immunohistochemical staining using rabbit polyclonal antibodies to each homologous protein localized native Hc-CBP-1 and Hc-CBP-2 to the brush borders or apical end of the intestinal epithelial cells (Fig. 4); however, anti-rHc-CBP-2 bound more intensely with the terminal web below the microvilli and to the hypodermis along the intestinal epithelial cell layer (Fig. 4b). No staining was observed in the adult worms when pre-immune rabbit serum was used (Fig. 4c).

\section{Overlapping peptide binding patterns of rHc-CBP-1 and rHc-CBP-2}

Peptide displays of rHc-CBP-1 and rHc-CBP-2 were screened with the homologous and heterologous rabbit antibodies and with $H$. contortus-infected sheep sera (Fig. 5). Given that both proteins are members of the cathepsin family and share sequence identity, some cross-reactivity was observed when each peptide display was screened with the heterologous antibodies; however, distinct immune reactivity profiles were also observed between each array and the homologous antisera. Figure $5 \mathrm{a}$ shows that the rHc-CBP-1 antibodies bound most strongly to peptides 1, 2, 3, 5-10, 26 and 28 in the homologous array where peptides $8-10$ were well recognized by antisera to both $\mathrm{rHc}-\mathrm{CBP}-1$ and rHc-CBP-2. rHc-CBP-1 peptides 19, 30, 34 and 39 showed strong binding to rabbit anti-rHc-CBP-2. In like manner, antiserum to rHc-CBP-2 bound most strongly to homologous rHc-CBP-2 peptides $1,2,5-11$, $25,28,29$ and 32 where rHc-CBP-2 peptides 8-11 cross-reacted with rabbit anti-rHc-CBP-1 (Fig. 5b). Serum from sheep infected with $H$. contortus reacted minimally with rHc-CBP-1 (peptides 11, 17 and 35) and rHc-CBP-2 (peptide 45). 


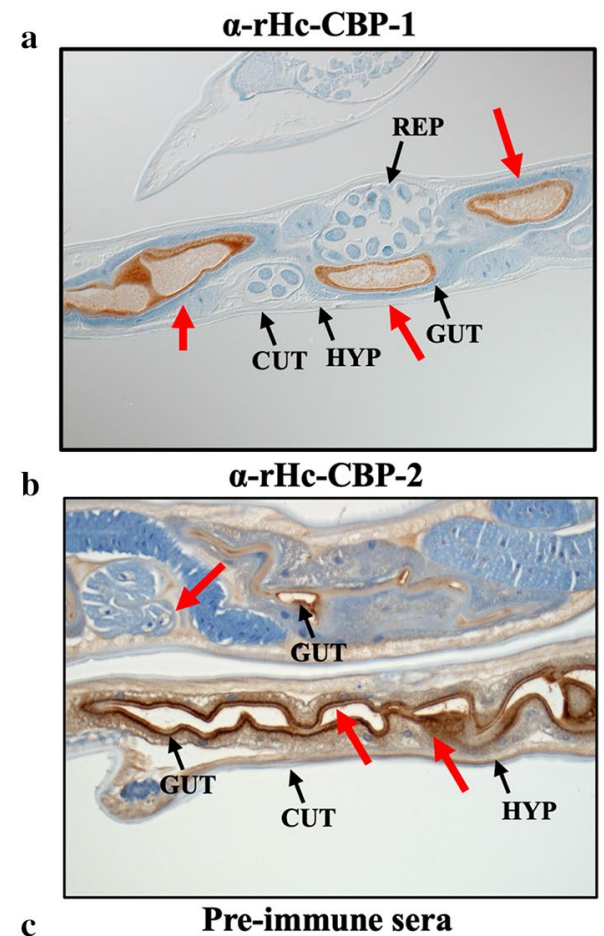

c

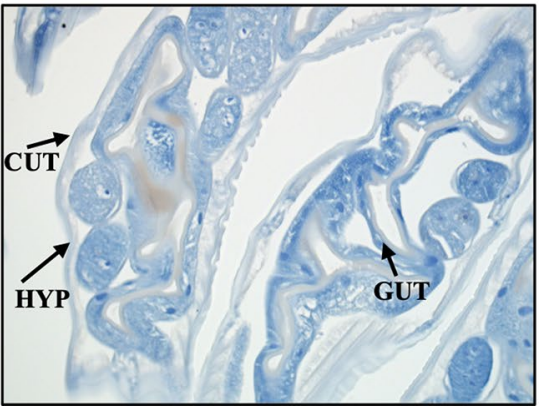

Fig. 4 Immunolocalization of native Hc-CBP-1 and $\mathrm{Hc}-\mathrm{CBP}-2$ in $\mathrm{H}$. contortus adults. Immunohistochemical staining of sectioned $\mathrm{H}$. contortus adult worms was performed using rabbit anti-a-rHc-CBP-1 (a), a-rHc-CBP-2 (b) antibody and pre-immune serum (c) (1:800 dilution). CUT = cuticle, GUT = gut, REP = reproductive organs, $\mathrm{HYP}=$ hypodermis. Magnification $=10 \times(\mathbf{a}) ; 20 \times(\mathbf{b}, \mathbf{c})$. Red arrows indicate the positive binding of a-rHc-CBP-1 or a-rHc-CBP-2 antibody to the tissues

\section{Transcriptional and immunological changes in Hc-cbp-1 and Hc-cbp-2 in immunosuppressed animals}

Transcript levels of Hc-cbp-1 and Hc-cbp-2 were evaluated in adult worms acquired from $\mathrm{Dex}^{+}$- and Dex ${ }^{-}$-treated animals (Fig. 6). As shown in Fig. 6a, the Hc- $c b p-1$ transcript encoding the parasite secreted protein was not affected by Dex ${ }^{+}$treatment of the host animals; however, the transcript level of Hc-cbp-2, where the cognate protein was not found in Hc-ESP, was nearly threefold higher in Dex ${ }^{+}$treated animals (Fig. 6b).

As shown in Fig. 7, there were also differences in the overall binding of sera from $\mathrm{Dex}^{+}$-treated animals to the peptide arrays. Based upon a two-tailed, pairwise $t$-test, numerous peptides from rHc-CBP-1 exhibited lower binding affinity to $\mathrm{Dex}^{+}$infected sera than to $\operatorname{Dex}^{-}$sera $(p<0.0001)$ suggesting suppressed immunity to this secreted antigen (Fig. 7a). Specifically, peptides $12,14,16,23,28,29,30$ and 37 showed a reduction in antibody titers compared to control animals. In contrast, peptides from the non-secreted Hc-CBP-2 did not show significant differences in antibody binding between $\operatorname{Dex}^{+}$and Dex ${ }^{-}$animals $(p=0.1078)$; only peptides 25 and 29 exhibited some variation in binding suggesting that the non-secreted Hc-CBP-2 is not affected by alterations in the host immune response (Fig. 7b).

\section{rHc-CBP-1 modulates co-stimulatory and pro-inflammatory cytokines}

Since Hc-CBP-1 is secreted, we examined the role of rHc-CBP-1 in modulating immune cells for transcriptional changes in three pro-inflammatory cytokines (TNF $\alpha$, IL-1 and IL-6) and three cell surface markers (CD40, CD80 and CD86) resulting from treatment of PBMCs from bovine blood with endotoxin-free rHc-CBP-1 (Fig. 8). As shown in Fig. 8a, TNF- $\alpha$ transcription was significantly downregulated in the pres-

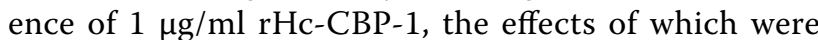
reversed in the presence of cathepsin $B$ inhibitor. This response appeared biphasic in that TNF- $\alpha$ expression did not change in the presence of 0.1 or $10 \mu \mathrm{g} / \mathrm{ml}$ rHc-CBP-1 (Fig. 8a). When the expression of IL-1 was examined (Fig. 8b), the data were inconclusive; rHcCBP-1 seemed only to suppress mRNA levels at the lowest concentration $(0.1 \mu \mathrm{g} / \mathrm{ml})$ where, in the presence of inhibitor, suppression returned to normal levels. At the intermediate and highest concentrations tested $(1.0-10 \mu \mathrm{g} / \mathrm{ml} \mathrm{rHc}-\mathrm{CBP}-1)$, mRNA values were unaffected except in the presence of inhibitor where mRNA expression values exceeded baseline levels; however, these responses were not statistically significant. When IL-6 was examined (Fig. 8c), transcription was downregulated at 0.1 and $1 \mu \mathrm{g} / \mathrm{ml} \mathrm{rHc}-\mathrm{CBP}-1$ with a trend toward control levels at the highest concentration of rHc-CBP-1. These effects were altered in a statistically significant manner in the presence of cathepsin $B$ inhibitor and became muted at higher concentrations of rHc-CBP-1 $(10 \mu \mathrm{g} / \mathrm{ml})$. 


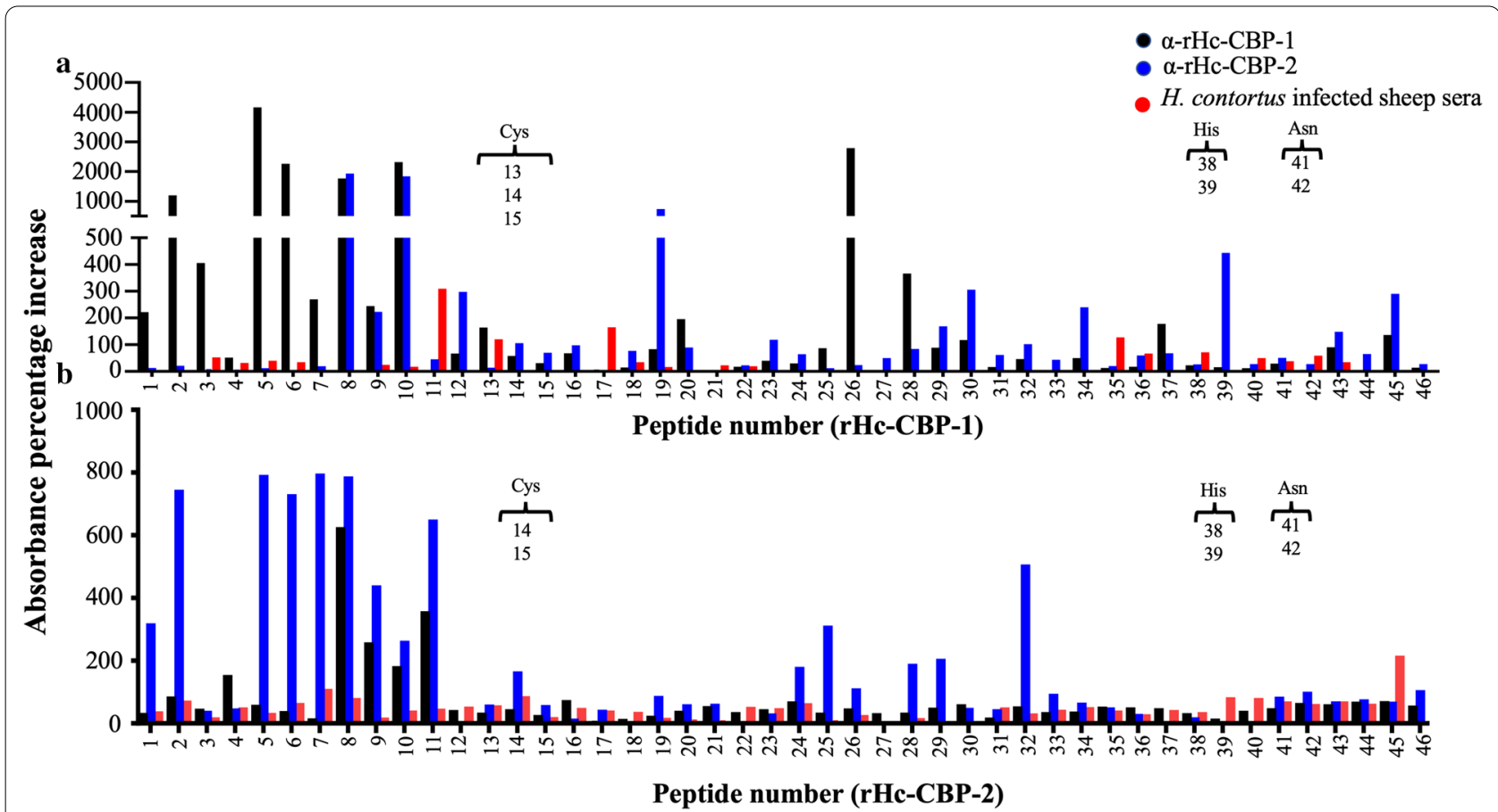

Fig. 5 Peptide arrays of Hc-CBP-1 and Hc-CBP-2 using homologous and heterologous antisera. Forty-six 15-amino-acid peptide with 8 amino acid overlaps among adjacent peptides were generated for Hc-CBP-1 (a) and Hc-CBP-2 (b). Antibody binding to each peptide was evaluated by ELISA at $410 \mathrm{~nm}$ using rabbit a-rHc-CBP-1 and a-rHc-CBP-2, and sheep $\mathrm{H}$. contortus-infected serum. Percent changes between immune and pre-immune sera $(\times 100)$ were plotted. Presence of active site is indicated in brackets. Cys =cysteine, His = histidine, Asn $=$ aspargine. The data are represented as means \pm standard error (SE); values where $p \leq 0.05$ were considered statistically significant

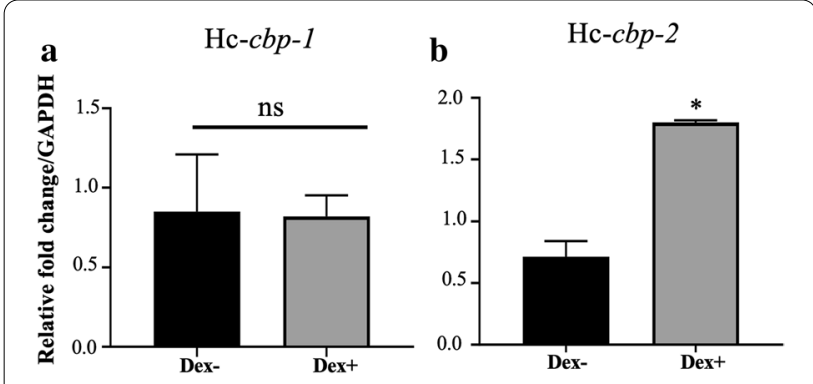

Fig. 6. Transcript abundance of Hc-cbp-1 and Hc-cbp-2 in Dex ${ }^{+}$- and Dex ${ }^{-}$-treated $H$. contortus-infected sheep. Relative transcript abundance for Hc-cbp-1 (a) and $\mathrm{Hc}-\mathrm{cbp}-2$ (b) was determined using cDNA synthesized from adult $H$. contortus total RNA obtained from $\mathrm{Dex}^{+}$and Dex ${ }^{-}$(control) animals and Hc-cbp-1 and Hc-cbp-2 specific qPCR primers. The values were normalized to adult $H$. contortus from Dex ${ }^{-}$animals and GAPDH was used as internal control. Data were analyzed using the $\triangle \triangle \mathrm{Ct}$ method where ${ }^{*} p \leq 0.05$. Data are reported as mean (M) of three replicates \pm SEM

With respect to the expression of co-stimulatory markers, Fig. 8d indicates that CD40 mRNA levels increased in a dose-dependent manner when cells were incubated in the presence of $\mathrm{rHc}-\mathrm{CBP}-1$, though the presence of inhibitor seemed not to alter this trend. The rHc-CBP-1 also upregulated the expression of CD80 where these effects were most pronounced at higher concentrations of $\mathrm{rHc}-\mathrm{CBP}-1(1 \mu \mathrm{g} / \mathrm{ml}$ and $10 \mu \mathrm{g} / \mathrm{ml})$ (Fig. 8e). As with $\mathrm{CD} 40$, these trends were not reversed in the presence of inhibitor. The effects of rHc-CBP-1 on CD86 expression (Fig. 8f) were consistent with a concentration-dependent downregulation of the marker. The effects of this downregulation were exacerbated rather than attenuated in the presence of inhibitor (Fig. 8f).

\section{Discussion}

Cathepsin B-like proteases are major virulence factors for helminth parasites and thus attractive therapeutic targets. Nematode cysteine proteases are often expressed in glands or intestinal cells and can act as key "digestive enzymes" [25]. Several studies have shown that helminth cathepsin B-like proteases can modulate host-pathogen interactions [39-41]. Our primary interest was to examine a subset of cysteine proteases in $H$. contortus that are shared among a subset of other clade $\mathrm{V}$ nematode parasites, in particular, blood-feeding nematodes. An in silico analysis of key datasets identified a family of genes linked to cathepsin B-like proteases, two of which encode proteases that harbor the classical Cys, His and Asn catalytic triad, suggesting that native Hc-CBP-1 and Hc-CBP-2 function as endopeptidases. 


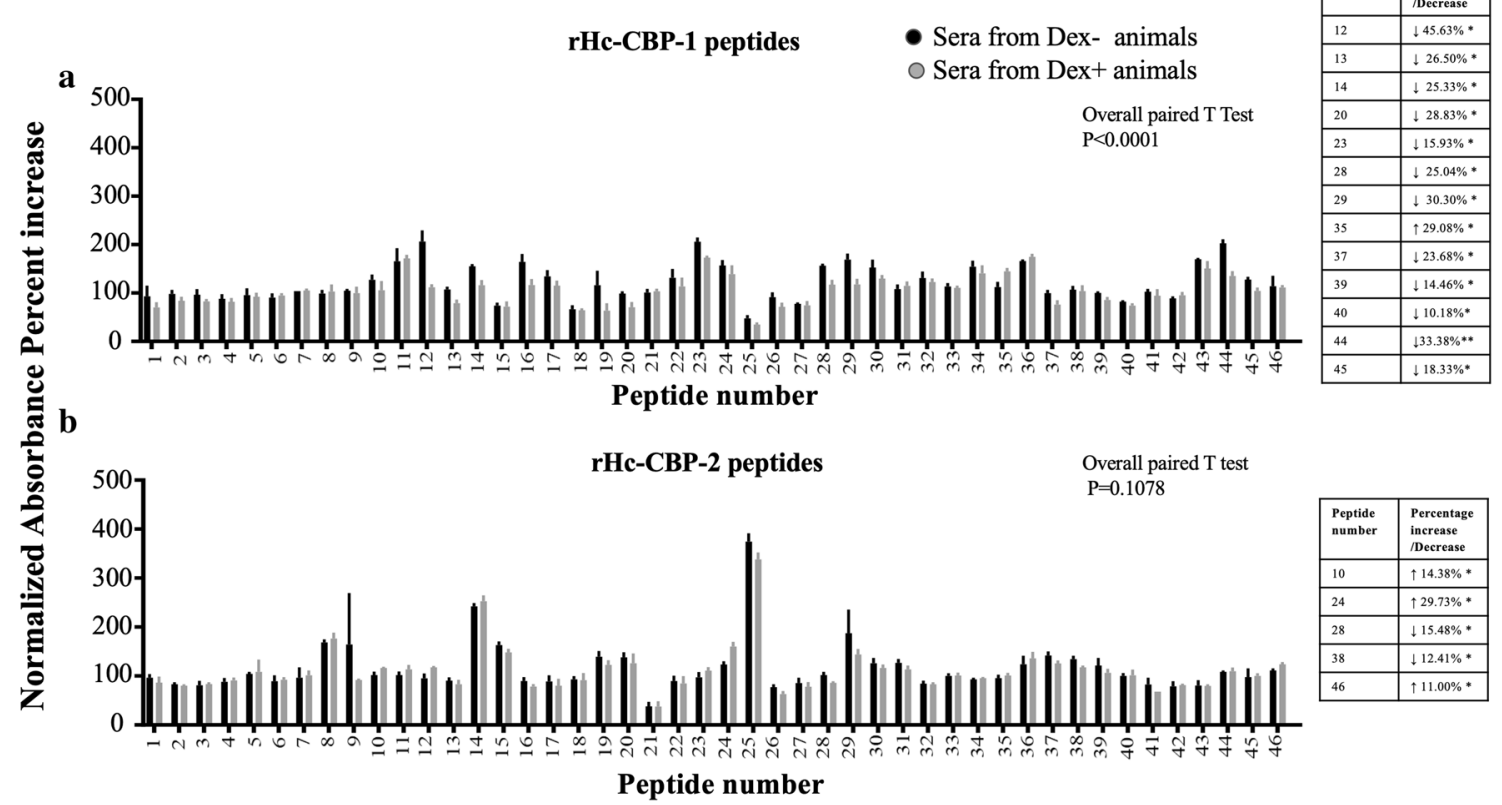

Fig. 7 Peptide arrays of Hc-CBP-1 and Hc-CBP-2 using sera from Dex ${ }^{+}$and Dex ${ }^{-}$H. contortus-infected sheep. Hc-CBP-1 (a) or Hc-CBP-2 (b) specific peptides (1-46) were incubated with serum obtained from Dex ${ }^{+}$and Dex ${ }^{-}$animals. Percent change between drug treated and non-drug treated $(\times 100)$ were plotted. The antibody binding percent signal intensities between animals were normalized by averaging the absorbance intensities of two animals/group using the formula: absorbance/mean absorbance of two animals $\times 100 \pm$ standard error (SE); values where $p \leq 0.05$ are considered statistically significant

In vitro proteolytic activity was not observed for either rHc-CBP using common host substrates, i.e. hemoglobin, IgGs or collagen (data not shown). Thus, native activities and substrates remain unresolved. It is possible that the proteins did not self-cleave to the active form in the presence of substrate. Alternatively, the lack of proper folding of the recombinant proteins and absence of post-translational modifications may also contribute to inactivity of $\mathrm{rHc}-\mathrm{CBP}-1$ and rHcCBP-2. However, both functionality and substrate affinity of these cathepsin-like proteins is continuing using a mammalian expression system to generate the recombinant proteins.

Native Hc-CBP-1 and Hc-CBP-2 are both present within the brush borders of the intestinal wall of the $H$. contortus adult worms where only Hc-CBP-1 was also found in adult Hc-ESPs. Additionally, Hc-CBP-2 was detected underneath the cuticle possibly in the hypodermis of adult worms and in additional brush borders of the intestinal wall. It is possible that Hc-CBP-2 is secreted in other stages of the parasite; however, this was not examined. Adult $H$. contortus cysteine proteases have been linked to the digestion of host blood; however, these were defined as cryptic proteins and not found in Hc-ESP [20].
Cysteine proteases in helminths have been shown to digest hemoglobin under the acidic conditions present in the parasite gut and food vacuoles [42]. According to the present study, however, Hc-CBP-1 is not a cryptic protein but is actively secreted into the worm's local environment and may help modulate at the host:parasite interface while in the abomasum where the L4 and adults feed. In contrast, amidst the presence of a secretory signal, Hc-CBP-2 was not secreted from the worm. However, caution must be taken when interpreting these data because active secretory signals define cellular events not parasitological activities. Namely, HcCBP-2 may indeed be secreted from the cell but not necessarily into the milieu that is transported outside the adult worm. Indeed, immunohistochemical staining showed Hc-CBP-2 was not confined to the parasite intestines, but was present throughout the hypodermis beneath the cuticle. This is consistent with regulating internal activities such as molting, cuticle remodeling and embryogenesis [26]. Also, Hc-CBP-2 may function as a cryptic parasite protein that interacts only with ingested host contents but remains attached to the worm's intestinal wall. Thus, it is unlikely Hc-CBP-1 and 


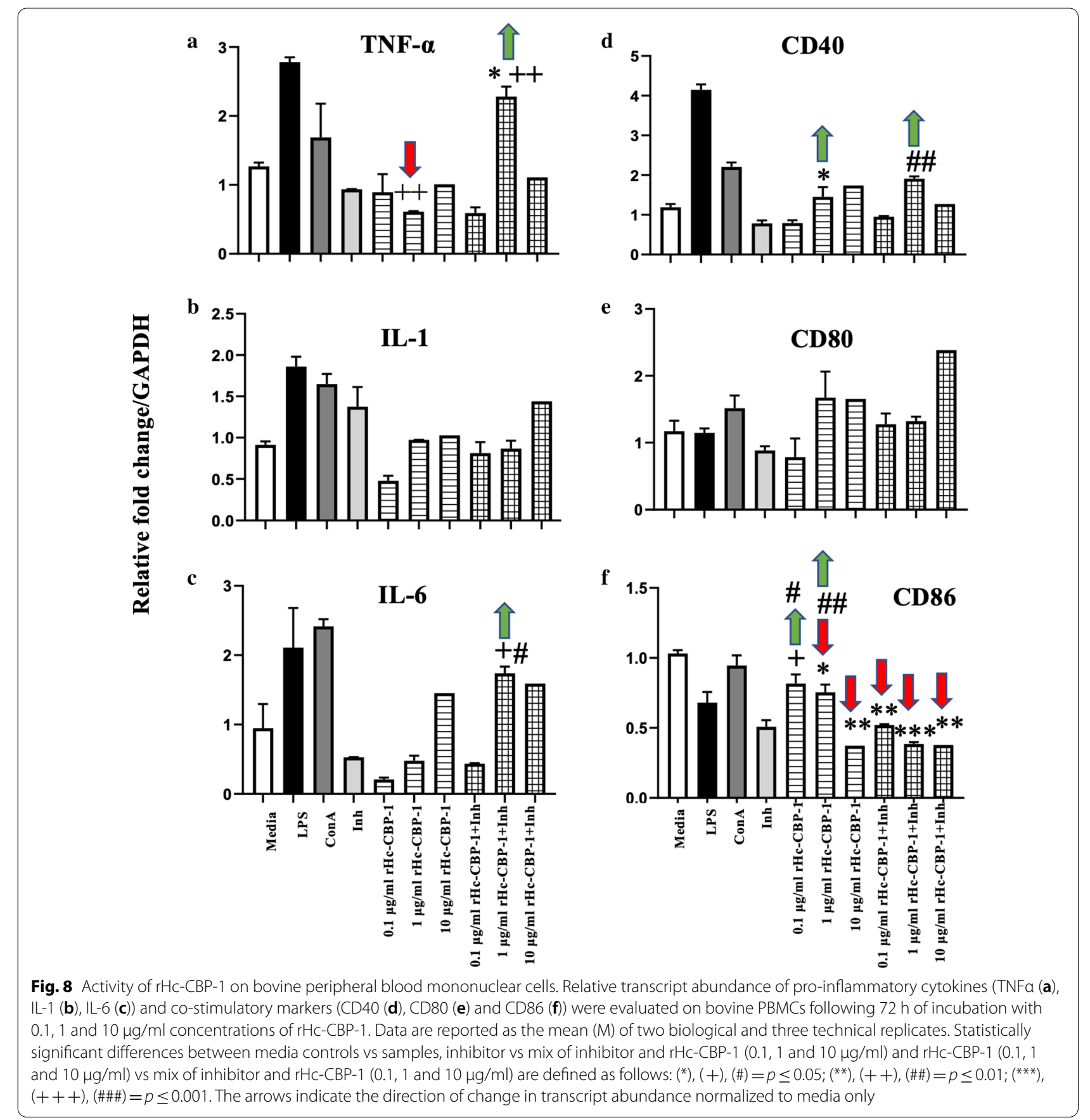

Hc-CBP-2 represent redundancies in functionality, and they may therefore differ in their natural substrates.

Mutagenesis and $\mathrm{pH}$ studies have demonstrated that the occluding loop of cathepsin B is a hydrogen ion-dependent gate for controlling not only the type of activity (exo- or endo-) but also maturation to the active state [43]. At low $\mathrm{pH}$ where activation is optimal, the occluding loop is thought to displace the
$\mathrm{N}$-terminal region of the protein, making it available for autoproteolysis. Once activated, cathepsin B functions as an exopeptidase at low $\mathrm{pH}$; however, cathepsin $\mathrm{B}$ proteases can also operate outside of the lysosomal fraction [44]. Hence, as demonstrated for other cathepsin B-like enzymes in $H$. contortus, Hc-CBP-1 and Hc-CBP-2 could function as both endo- and exopeptidases. 
The use of peptide arrays screened with antisera can be effective for identifying immunodominant epitopes and developing subunit vaccines [45, 46]. Herein, we characterized and compared antibody epitopes among rHc-CBP-1 and rHc-CBP-2 using both homologous and heterologous rabbit polyclonal antibodies and sheep infection serum. The observed immunodominant peptides were relegated to the exposed $\mathrm{N}$-terminal loop region of $\mathrm{rHc}-\mathrm{CBP}-1$ and $\mathrm{rHc}-\mathrm{CBP}-2$ and less so to the internally located active sites which are folded inward. In our analysis, we also found that peptides 8, 9, 10 and 11 bound to both homologous and heterologous antisera. Not surprisingly, the overall immune profiles generated using rabbit antisera to $\mathrm{rHc}-\mathrm{CBP}-1$ and $\mathrm{rHc}-\mathrm{CBP}-2$ and the sheep sera from experimentally infected animals were not congruent where rHc-CBP-1 and rHc-CBP-2 were poorly recognized by the sheep sera. Although this is predictable with the cryptic Hc-CBP-2, it was less so with the secreted Hc-CBP-1. Amidst a 57\% sequence similarity among the two Hc-CBP, the peptide profiles for the homologous and heterologous rabbit antibodies were relatively distinct, further supporting a lack of redundancy in substrate activities among these cathepsins [47]. However, based upon these arrays, the few areas of overlap in antibody binding may still afford targets for the development of conserved subunit vaccines.

Dexamethasone is an immune-suppressive drug that is widely used to treat immune conditions in sickened animals; however, it has also been used to investigate hostparasite relationships [48-50]. Surprisingly, the transcript levels of the secreted Hc-cbp-1 did not change in $\mathrm{Dex}^{+}$ animals whereas the non-secreted, Hc-cbp-2 transcript increased significantly. This suggests that production and therefore secretion of Hc-CBP-1 is little effected by the immune status of the host and that the non-secreted HcCBP-2, likely a cryptic protein, must interact directly with ingested host contents because it appears downregulated or controlled by a healthy immune system. In agreement with the changes in transcription, host antibody levels to Hc-CBP-2, which is attached to the parasite intestinal wall, were slightly higher in $\mathrm{Dex}^{+}$animals relative to controls, which is perplexing. Native Hc-CBP-2 does possess a secretory signal, and its secretion may be related to the immune status of the host, which could explain why transcription levels increased in immunocompromised animals. Others who have studied dexamethasone treatment in sheep infected with Haemonchus demonstrated overall lower antibody responses relative to non-treated animals [48].

Haemonchus contortus L4s and adults feed on blood for nutrients; however, host blood also contains immune factors that the parasite must circumvent to maintain blood flow and its own survival. Host PBMCs contain monocytes, B cells, $\mathrm{T}$ cells, dendritic cells and macrophages [51], the collection of which can induce Th1 responses that drive inflammation and Th2 responses that resolve inflammation. Typically, Th1 responses are characterized by changes in TNF $\alpha$, INF $\gamma$, nitric oxide, IL-1 and IL-6, which are important in promoting and maintaining proinflammatory responses during infection. Inflammation-related responses can also be monitored by examining cell activation via cell surface markers. One such co-stimulatory marker, CD40, is a $50-\mathrm{kDa}$ molecule expressed on different activated cell types, including monocyte macrophages [52]. Two other surface molecules, CD80 and CD86, are also important in immune cell activation in response to pathogens [53-55] and associated with inflammation. On this basis, our data suggest that the secreted rHc-CBP-1 downregulates TNF $\alpha$, IL- 1 and IL-6 transcripts in host PBMCs inhibiting the host Th1 environment. In contrast, we also found that rHc-CBP-1 induces significant expression of CD40 and suppression of CD86, indicating a mixed response. Recently, Chen et al. showed that recombinant cathepsin B from Fasciola gigantica induces PBMC cell cytokine profiles consistent with an active role in parasitism and host control [56]. Taken together, these data are consistent with Hc-CBP-1 immunomodulating the host inflammatory cells to advance its survival and growth. More detailed mechanisms involving key molecules of apoptosis, cell proliferation and signaling molecules merit further investigation.

In summary, we have described two $H$. contortus-derived cathepsin B-like proteases that show sequence similarity to proteins in other clade $\mathrm{V}$ parasite nematodes. Although both were selected based upon the presence of putative secretory signals, and both were found in the exposed layers of the intestinal epithelium, only Hc-CBP-1 was present in Hc-ESP of adult worms. Peptide arrays demonstrated regions comprising both specific and overlapping antibody binding between the two cathepsins. The greatest cross reactivity occurred predominantly within the $\mathrm{N}$-terminal region of the mature proteins where $\mathrm{N}$-linked glycosylation and the formation of an active protease originate. This suggests that pan-specific antibodies can be developed against this region that in turn may prevent the formation of an active protein. It is interesting to note that rabbit antibodies bound very strongly to the $\mathrm{N}$-terminal region of both rHc-CBP-1 and rHc-CBP-2, which were largely unrecognized by sheep infection serum, suggesting that natural immunity is lacking. We also show that rHc-CBP-1 modulates pro- and anti-inflammatory responses within the myriad of cells that make up PBMCs and that responses to both these proteins are affected by the immune status of the host suggesting again that they are viable targets for immune intervention against $H$. contortus. 


\section{Supplementary Information}

The online version contains supplementary material available at https://doi. org/10.1186/s13071-021-05010-y.

Additional file 1: Dataset S1. H. contortus protein sequences retrieved from MEROPS and NCBI databases.

Additional file 2: Dataset S2. H. contortus cysteine and aspartate proteases that were less than $60 \%$ similar to peptidases from P. pacificus, C. elegans and 0 . aries.

Additional file 3: Dataset S3. H. contortus cysteine and aspartate proteases that were greater than $60 \%$ similar to peptidases from T. circumcincta, A. caninum and N. americanus.

Additional file 4: Dataset S4. Selected H. contortus cysteine and aspartate proteases that are in common with peptidases from T. circumcincta, A. caninum and $N$. americanus.

Additional file 5: Dataset S5. Conserved domain search of selected $H$. contortus cysteine and aspartate proteases.

Additional file 6: Dataset S6. Overlapping peptides derived from HcCBP-1 and HC-CBP-2 protein sequences.

Additional file 7: Figure S1. Recombinant protein expression of $\mathrm{Hc}-\mathrm{CBP}-1$ and $\mathrm{Hc}-\mathrm{CBP}-2$. Recombinant proteins $\mathrm{rHc}-\mathrm{CBP}-1$ (A) and rHc-CBP-2 (B) were eluted in $15 \mathrm{mM}$ to $400 \mathrm{mM}$ imidazole, separated on SurePAGE ${ }^{\text {TM }}$ (Genscript, Piscataway, NJ) 4-20\% BIS-TRIS gradient gel and then stained with Coomassie blue.

\section{Acknowledgements}

We gratefully acknowledge Ms. Deborah Hebert, Ms. Marsha Campbell and members of Research Animal Services at the Beltsville Agricultural Research Centre for their technical assistance.

\section{Authors' contributions}

MB, DZ, WT, AR developed the concept and the design of the study. DZ supervised the study. MB and DZ performed the laboratory assays and data analyses. MB and DZ drafted the manuscript. All authors contributed to the draft review. All authors read and approved the final manuscript.

\section{Funding}

This research is supported by USDA Research Project \#8042 32000105 00D to DZ and WT and NICHD R01 HD099072 to AR.

\section{Availability of data and materials}

Data available on request from the authors.

\section{Declarations}

\section{Ethics approval and consent to participate}

All experimental protocols involving animals were approved by the Beltsville Animal Care and Use Committee and performed in accordance with relevant guidelines and regulations.

\section{Consent for publication}

Not applicable.

\section{Competing interests}

Authors declare no competing interests.

\section{Author details}

${ }^{1}$ Animal Parasitic Diseases Laboratory, USDA-ARS, Beltsville, MD, USA. ${ }^{2} \mathrm{O} a \mathrm{k}$ Ridge Institute for Science and Education, Oak Ridge, Tennessee, USA. ${ }^{3}$ University of Massachusetts Medical School, University of Massachusetts, Worcester, MA, USA.

Received: 21 June 2021 Accepted: 9 September 2021

Published online: 19 November 2021
References

1. Perry BD. Investing in animal health research to alleviate poverty: ILRI (aka ILCA and ILRAD): 2002.

2. Prichard R. Genetic variability following selection of Haemonchus contortus with anthelmintics. Trends Parasitol. 2001;17(9):445-53.

3. Clark CH, Kiesel GK, Goby CH. Measurement of blood loss caused by Haemonchus contortus. Am J Vet Med. 1962:23:977-80.

4. Kotze A, Prichard R. Anthelmintic resistance in Haemonchus contortus: history, mechanisms and diagnosis. Adv Parasitol. 2016;93:397-428.

5. Lightowlers M, Rickard M. Excretory-secretory products of helminth parasites: effects on host immune responses. Parasitology. 1988;96(S1):S123-66.

6. Smith W. Prospects for vaccines of helminth parasites of grazing ruminants. Int J Parasitol. 1999:29(1):17-24.

7. Xu J, Liu RD, Bai SJ, Hao HN, Yue WW, Xu YX, Long SR, Cui J, Wang ZQ. Molecular characterization of a Trichinella spiralis aspartic protease and its facilitation role in larval invasion of host intestinal epithelial cells. PLoS Negl Trop Dis. 2020;14(4):e0008269.

8. Tuo W, Li L, Lv Y, Carrillo J, Brown D, Davis WC, et al. Abomasal mucosal immune responses of cattle with limited or continuous exposure to pasture-borne gastrointestinal nematode parasite infection. Vet Parasitol. 2016;229:118-25.

9. Hepworth MR, Maurer M, Hartmann S. Regulation of type 2 immunity to helminths by mast cells. Gut microbes. 2012;3(5):476-81.

10. King IL, Mohrs K, Meli AP, Downey J, Lanthier P, Tzelepis F, et al. Intestinal helminth infection impacts the systemic distribution and function of the naive lymphocyte pool. Mucosal Immunol. 2017;10(5):1160-8.

11. MacDonald AS, Araujo MI, Pearce EJ. Immunology of parasitic helminth infections. Infect Immun. 2002;70(2):427-33.

12. Anthony RM, Rutitzky LI, Urban JF, Stadecker MJ, Gause WC. Protective immune mechanisms in helminth infection. Nat Rev Immunol. 2007;7(12):975-87.

13. Meeusen ENT, Balic A. Do eosinophils have a role in the killing of helminth parasites? Parasitol Today. 2000;16(3):95-101.

14. Huang $L$, Appleton JA. Eosinophils in helminth infection: defenders and dupes. Trends Parasitol. 2016;32(10):798-807.

15. Jasmer DP, Perryman LE, Conder GA, Crow S, McGuire T. Protective immunity to Haemonchus contortus induced by immunoaffinity isolated antigens that share a phylogenetically conserved carbohydrate gut surface epitope. J Immunol. 1993;151(10):5450-60.

16. Schallig TS, Van Leeuwen MA, Cornelissen AW. Protective immunity induced by vaccination with two Haemonchus contortus excretory secretory proteins in sheep. Parasite Immunol. 1997;19(10):447-53.

17. Smith TS, Graham M, Munn EA, Newton SE, Knox DP, Coadwell WJ, McMichael-Phillips D, Smith H, Smith WD, Oliver JJ. Cloning and characterization of a microsomal aminopeptidase from the intestine of the nematode Haemonchus contortus. Biochem Biophys Acta. 1997;1338(2):295-306.

18. Yatsuda AP, Krijgsveld J, Cornelissen AW, Heck AJ, de Vries E. Comprehensive analysis of the secreted proteins of the parasite Haemonchus contortus reveals extensive sequence variation and differential immune recognition. J Biol Chem. 2003;278(19):16941-51.

19. Cachat E, Newlands G, Ekoja S, McAllister H, Smith W. Attempts to immunize sheep against Haemonchus contortus using a cocktail of recombinant proteases derived from the protective antigen. H-gal-GP Parasite Immunol. 2010;32(6):414-9.

20. Willadsen P, Riding GA, McKenna RV, Kemp DH, Tellam RL, Nielsen $J \mathrm{~N}$, et al. Immunologic control of a parasitic arthropod Identification of a protective antigen from Boophilus microplus. J Immunol. 1989:143:1346-51.

21. Jasmer DP, McGuire TC. Protective immunity to a blood-feeding nematode (Haemonchus contortus) induced by parasite gut antigens. Infect Immun. 1991;59(12):4412-7.

22. Munn EA, Smith TS, Graham M, Tavernor AS, Greenwood CA. The potential value of integral membrane proteins in the vaccination of lambs against Haemonchus conortus. Int J Parasitol. 1993;23(2):261-9.

23. Smith W, Smith S, Murray J. Protection studies with integral membrane fractions of Haemonchus contortus. Parasite Immunol. 1994;16(5):231-41. 
24. Nisbet A, Meeusen E, González J, Piedrafita D. Immunity to Haemonchus contortus and vaccine development. Adv Parasitol. 2016:93:353-96.

25. Williamson AL, Brindley PJ, Knox DP, Hotez PJ, Loukas A. Digestive proteases of blood-feeding nematodes. Trends Parasitol. 2003;19(9):417-23.

26. Grote A, Caffrey CR, Rebello KM, Smith D, Dalton JP, Lustigman S. Cysteine proteases during larval migration and development of helminths in their final host. PLoS Negl Trop Dis. 2018;12(8):e0005919.

27. Zhu X, Chen D, Tian A, Hou J, Li J, Tian X, et al. The multitasking Fasciola gigantica cathepsin $B$ interferes with various functions of goat peripheral blood mononuclear cells in vitro. Front Immunol. 2019;10:1707.

28. Soloviova K, Fox EC, Dalton JP, Caffrey CR, Davies SJ. A secreted schistosome cathepsin B1 cysteine protease and acute schistosome infection induce a transient T helper 17 response. PLoS Negl Trop Dis. 2019;13(1):e0007070.

29. Lu S, Wang J, Chitsaz F, Derbyshire MK, Geer RC, Gonzales NR, Gwadz M, Hurwitz DI, Marchler GH, Song JS, Thanki N. CDD/SPARCLE: the conserved domain database in 020. Nucleic Acids Res. 2020:48(D1):D265-8.

30. Tuo W, Zarlenga D, Hebert D, Miramontes E, Fetterer R. A two-step method to generate highly-purified nematode eggs from feces: sucrose flotation followed by density gradient centrifugation using lymphocyte separation medium. Comp Parasitol. 2015;82:275-9.

31. Bakshi M, Hebert D, Gulbronson C, Bauchan G, Tuo W, Zarlenga D. Ostertagia ostertagi mediates early host immune responses via macrophage and Toll like receptor pathways. Infect Immun. 2021. https:// doi.org/10.1128/IAl.00017-21.

32. Adams DB, Lynch JJ, Anderson BH, Fell LR, Hinch GN, Munro RK. The intensity of resistance by mature Merino ewes against Haemonchus contortus and Tichostrongylus colubriformis in single-species and combined-species infection. Aust Vet J. 1990;67:443-5.

33. Waterhouse A, Bertoni M, Bienert S, Studer G, Tauriello G, Gumienny R, Heer FT, de Beer TA, Rempfer C, Bordoli L, Lepore R. SWISS-MODEL: homology modelling of protein structures and complexes. Nucleic Acids Res. 2018:46(W1):W296-303.

34. Benkert P, Künzli M, Schwede T. QMEAN server for protein model quality estimation. Nucleic Acids Res. 2009;37:W510-4.

35. Benkert P, Biasini M, Schwede T. Toward the estimation of the absolute quality of individual protein structure models. Bioinformatics. 2011;27:343-50.

36. Livak KJ, Schmittgen TD. Analysis of relative gene expression data using real-time quantitative PCR and the 2deltadeltaCT method. Methods. 2001;25(4):402-8

37. Lecová L, Růžičková M, Laing R, Vogel H, Szotáková B, Prchal L, Lamka J, Vokřál I, Skálová L, Matoušková P. Reliable reference gene selection for quantitative real time PCR in Haemonchus contortus. Mol Biochem Parasitol. 2015;201(2):123-7.

38. Waterhouse A, Bertoni M, Bienert S, Studer G, Tauriello G, Gumienny R, Heer FT, de Beer TAP, Rempfer C, Bordoli L, Lepore R, Schwede T. SWISSMODEL: homology modelling of protein structures and complexes. Nucleic Acids Res. 2018;46:W296-303.

39. Harrop SA, Sawangjaroen N, Prociv P, Brindley PJ. Characterization and localization of cathepsin B proteinases expressed by adult Ancylostoma caninum hookworms. Mol Biochem Parasitol. 1995;71(2):163-71.

40. Ricciardi A, Visitsunthorn K, Dalton JP, Ndao M. A vaccine consisting of Schistosoma mansoni cathepsin B formulated in Montanide ISA 720 VG induces high level protection against murine schistosomiasis. BMC Infect Dis. 2016;16:112.
41. Han Y, Yue X, Hu CX, Liu F, Liu RD, He MM, et al Interaction of a Trichinella spiralis cathepsin B with enterocytes promotes the larval intrusion into the cells. Res Vet Sci. 2020;130:110-7.

42. Caffrey CR, Goupil L, Rebello KM, Dalton JP, Smith D. Cysteine proteases as digestive enzymes in parasitic helminths. PLoS Negl Trop Dis. 2018;12:e0005840

43. Cathers BE, Barrett C, Palmer JT, Rydzewski RM. pH Dependence of inhibitors targeting the occluding loop of cathepsin B. Bioorg Chem. 2002:30(4):264-75.

44. Giusti I, D'Ascenzo S, Millimaggi D, Taraboletti G, Carta G, Franceschin $\mathrm{N}$, et al. Cathepsin $\mathrm{B}$ mediates the $\mathrm{pH}$-dependent proinvasive activity of tumor-shed microvesicles. Neoplasia. 2008;10(5):481-8.

45. Soria-Guerra RE, Nieto-Gomez R, Govea-Alonso DO, Rosales-Mendoza S. An overview of bioinformatics tools for epitope prediction: implications on vaccine development. J Biomed Inform. 2015;53:405-14.

46. De Groot AS, Moise L, McMurry JA, Martin W. Epitope-based immunome-derived vaccines: a strategy for improved design and safety. Clin Appl Immunomics. 2009;88:39-69.

47. Nägler DK, Ménard R. Family C1 cysteine proteases: biological diversity or redundancy? Biol Chem. 2003;384(6):837-43.

48. Pena M, Miller J, Horohov D. Effect of dexamethasone treatment on the immune response of Gulf Coast Native lambs to Haemonchus contortus infection. Vet Parasitol. 2004;119(2-3):223-35.

49. Presson B, Gray G, Burgess S. The effect of immunosuppression with dexamethasone on Haemonchus contortus infections in genetically resistant Merino sheep. Parasite Immunol. 1988;10(6):675-80.

50. Adams DB. Investigation with dexamethasone of the processes which moderate immunity against the nematode, Haemonchus contortus, in sheep. Aust J Exp Biol Med Sci. 1983;61(3):345-53.

51. Kleiveland CR. Peripheral blood mononuclear cells. The impact of food bioactives on health. 2015. p 161-7.

52. Alderson MR, Armitage RJ, Tough TW, Strockbine L, Fanslow WC, Spriggs MK. CD40 expression by human monocytes: regulation by cytokines and activation of monocytes by the ligand for CD40. J Exp Med. 1993:178(2):669-74.

53. Kiener PA, Moran-Davis P, Rankin BM, Wahl AF, Aruffo A, Hollenbaugh D. Stimulation of CD40 with purified soluble gp39 induces proinflammatory responses in human monocytes. J Immunol. 1995; 155(10):4917-25.

54. O'Sullivan B, Thomas R. CD40 and dendritic cell function. Crit Rev Immunol. 2003;23:83-107.

55. Brossart P, Grünebach F, Stuhler G, Reichardt VL, Möhle R, Kanz L, et al. Generation of functional human dendritic cells from adherent peripheral blood monocytes by CD40 ligation in the absence of granulocytemacrophage colony-stimulating factor. Blood J Am Soc Hematol. 1998:92(11):4238-47

56. Chen D, Tian A, Hou J, Li J, Tian X, Yuan X, et al. The multitasking Fasciola gigantica cathepsin $B$ interferes with various functions of goat peripheral blood mononuclear cells in vitro. Front Immunol. 2019:10:1707.

\section{Publisher's Note}

Springer Nature remains neutral with regard to jurisdictional claims in published maps and institutional affiliations. 